\title{
Trepanning Optical System Using Tilting Focus Lens for Laser Drilling Process
}

\author{
Jiwhan Noh*, Jaehoon Lee*, Jeong Suh*,and Suckjoo Na** \\ * KIMM (Korea Institute of machinery \&materials) \\ 104 Sinseongno,Yuseong-gu,Daejeon,Korea \\ Email address:njw733@kimm.re.kr \\ ** KAIST(Korea Advanced Institute of Science and Technology) \\ 371-1 Guseong-dong, Yuseong-gu, Daejeon, Korea \\ Email address: sjna@kaist.ac.kr
}

\begin{abstract}
Trepanning optics is used for laser drilling process. It enables more precise drilling with fewer heat affected zone. To actually use the trepanning optical module for the laser drilling manufacturing system, it must first be small and light. This paper proposes trepanning optical system using tilting focus lens to ensure the small and light form factor of the trepanning optical system. The size of trepanning could be significantly reduced by removing two wedges from the conventional wedge system. Optical design was completed using optical laser tracing, and the trepanning optical system which was based on the optical design was produced. Drilling processing of 400um thickness steel was performed using the trepanning optics system.
\end{abstract}

DOI: $10.2961 /$ jlmn.2009.03.0019

Keywords: trepanning optics, laser ablation drilling process, tilting focusing lens.

\section{Introduction}

Mechanical drill is generally used to make a hole on a material. However, many studies are ongoing on laser drilling because of limitations of mechanical drill $[1,2,3,4,5]$. The unobtrusive drilling method of focusing the laser beam to the material enables drilling of holes as small as tens or hundreds microns in diameter. Laser beam can drill holes on very hard and soft materials that are difficult with mechanical equipment. $\mathrm{CO}_{2}$ laser, $\mathrm{Nd}$ :YAG laser or eximer laser is used for laser drilling. Especially to drill the hold with clean surface, ultra-short pulse laser (femtosecond laser or picosecond laser) is used $[6,7]$. Using the ultrashort pulse laser can improve the drilling quality as it can reduce melting zone generated when using the conventional laser. There are on-going studies of laser drilling processes to improve the drilling quality without changing the laser source. Various laser drilling processes include single pulse drilling, percussion drilling and trepanning drilling. Of these, trepanning drilling is known to have better drilling properties [8]. Single pulse drilling is a method of making holes with a laser pulse and thus requires large pulse energy. Since it is processed with a single pulse, its drilling process time is very short but generates high material melting due to large pulse energy and thus greatly degrades drilling quality. Percussion drilling makes holes by irradiating multiple pulse beams on one location. Although this method induces less material melting than single pulse drilling, it still generates melting zones and is impossible to cleanly drill. Furthermore, when using Gaussian laser beam, it generates tapered drilling. Because of the taper, it is impossible to drill with high aspect ratio. Instability of laser source power also degrades repeatability of drilling diameter when the percussion drilling process is used. Trepanning drilling is the method of drilling while rotating the focus beam at high speed using the optical device. The in- cident beam is irradiated on the specimen with inclined angle at the same time as the focused beam is rotated. Another function of trepanning optics is that the polarized light can be rotated to the same direction toward the drilling cross section surface. This method can drill larger hole than focused beam but drastically reduces the melting zone around the drilled area. Reverse tapered drilling is also possible when irradiating the focused laser beam with inclined angle. Reverse tapered drilling means that the diameter of drilling entrance point is smaller than the exit point. The drilling speed can be increased by irradiating the focused beam because the focused beam is irradiated with inclined angle [9].

By rotating the polarized light in the same direction toward the drilling cross section surface, the drilled surface can be smoothed uniformly. As the reflection rate differs according to the polarized light direction, the asymmetry of drilling exit can also be removed by rotating the polarized light.

For trepanning drilling, trepanning optics to rotate the beam is needed or the drilled material itself can be rotated. Rotating the drilled material has the constraint of the material must be small since it is moved. Using trepanning optics does not require rotation of the material. Trepanning optics can be structured in one of the 4 ways: 1) rotating the lens to eccentricity, 2) using the mirror scanner, 3) using the dove prism, and 4) using the wedge. Methods of using the lens eccentricity and dove prism can make it easy to structure the optical system but difficult to rotate the beam in high speed because of the eccentricity of lens or dove prism. They are also difficult to control the angle of beam irradiated to the drilled material. Using the 2-axis mirror scanner makes it difficult to drill a small hole and impossible to control the angle of beam irradiated to the drilled material. Using the wedge makes it easier to rotate 
the beam in high speed than other methods. It is also possible to control the incident angle of beam irradiated to the drilled material $[10,11]$.

Therefore, this paper will discuss the result of laser drilling using the trepanning optics with wedge. The existing trepanning optics using the wedge is described, and the new improved method is proposed. The problem with the existing trepanning optics was that it was very difficult to mount the optic module to the drill manufacturing system because of trepanning module's large size and heavy. The trepanning optic module may have to be moved in case of actual laser drill manufacturing system. There can be a overloaded burden to the drill manufacturing system if the optic module is large and heavy. In order to apply the trepanning optics to laser drill manufacturing system trepanning optics size should be under $50 \mathrm{~cm}$ and trepanning optics weight should be under $25 \mathrm{~kg}$. of course this value can be changed according to drilling manufacturing system. Therefore, this paper proposes a new type of optics to reduce the size and weight of trepanning optics. The optical system was designed, and trepanning optics was manufactured using it. Then the drilling test was conducted using the trepanning optical system.

\section{Experimental method}

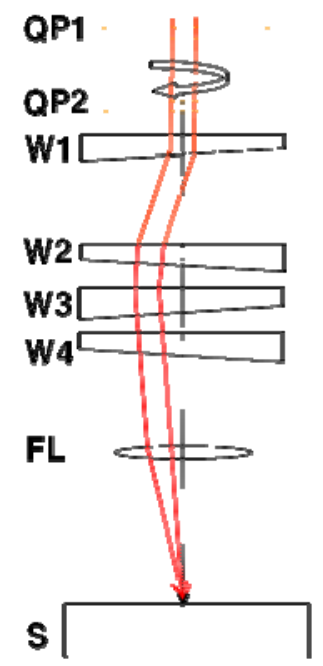

(a)

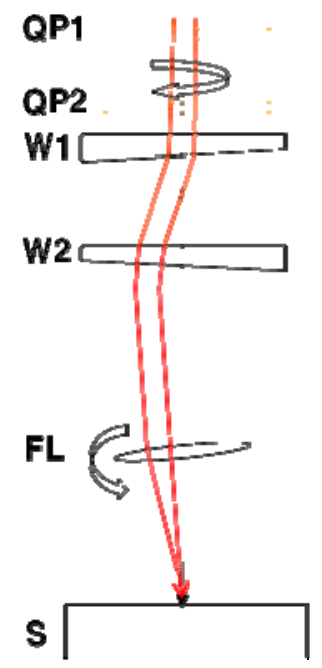

(b)
Figure 1. (a) the conventional wedge trepanning optics. (b) the new type of wedge trepanning optics ( QP : quarter wave plate, $\mathrm{W}$ : wedge, $\mathrm{FL}$ : focus lens, $\mathrm{S}$ : specimen)

Figure 1(a) shows the conventional method of wedge trepanning optics. Wedges 1 and 2 are installed at $180^{\circ}$ rotated to each other. Therefore, there is an offset of the laser beam to the optical axis as it passes through the wedge 1 and wedge 2 . When the wedges 1 and 2 are rotated and the beam passes through the focus lens in that condition, the laser beam is irradiated to the specimen with inclined angle and rotates on specimen. Even when the wedge 1 and wedge 2 are supposed to be the same wedge angle there is still the error of around $0.2^{\circ}$ because of manufacturing error. Wedges 3 and 4 are used to correct the error generated by the wedges 1 and 2. The wedges 3 and 4 are designed to have much smaller angle than wedges 1 and 2 . To rotate the polarized light direction, quarterwave plates 1 and 2 are used. Let us assume that the linearly polarized beam is generated by the laser source. Then the linearly polarized laser beam is converted to circularly polarized light as it passes through the quarterwave plate 1 . It is then converted back to the linearly polarized laser beam when passing through the quarterwave plate 2 . When quarterwave plate 2 , wedge 1 , wedge 2 , wedge 3 and wedge 4 all rotate, the polarized light also rotates as the laser beam rotates.

Figure 1 (b) shows the new trepanning optical system proposed in this paper. It uses tilting of focus lens instead of wedge 3 and wedge 4 in Figure 1 (a). By removing wedges 3 and 4 , the overall trepanning size and weight can be reduced. Small and light trepanning optical system is required when the system must be moved in actual drilling system.

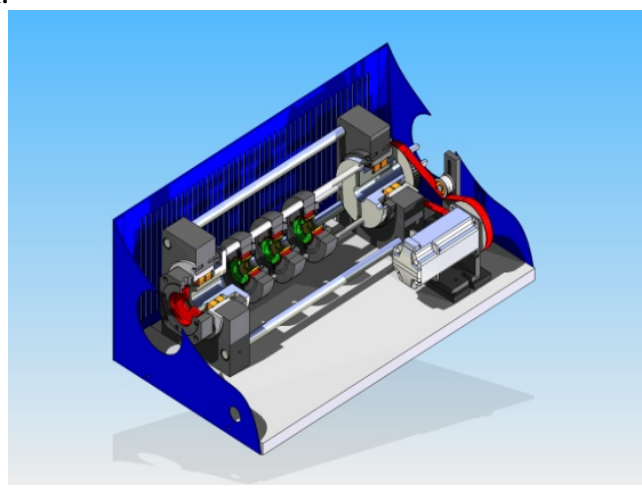

(a)

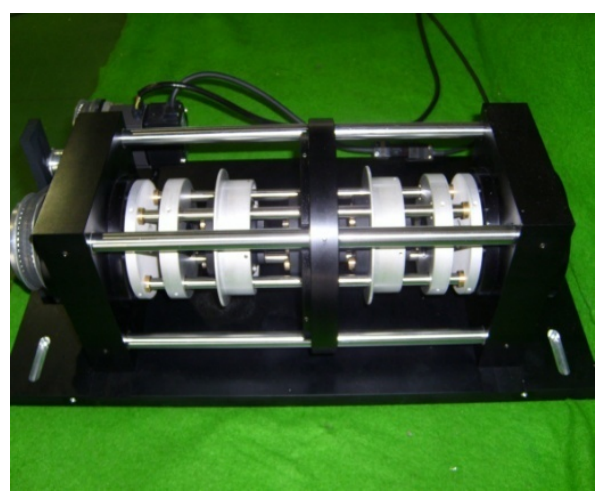

(b)

Figure2. (a) the mechanical structure of repanning optics.

(b) Photography of trepanning optics.

Figure 2 (a) shows the mechanical structure CAD design of trepanning optics, and Figure 2 (b) shows photography of trepanning optics. The mechanical structure that holds the wedge and focusing lens is rotated using a belt type motor. The rotation speed can reach as high as 3000rpm. An AC servo motor (MITSUBISHI HC-KFS23) is used. $118 \mathrm{~V}$ and $1.1 \mathrm{~A}$ power is supplied to the motor. The motor is rotated at the specified rotation speed using the motor controller. The trepanning optics size is $50 \mathrm{~cm} * 20$ $\mathrm{cm}$ and trepanning optics weight is $25 \mathrm{~kg}$. Different inclination angles and different size drilling are possible by changing the wedge and focusing lens. E43-650 wedge from Edmund is used. It is made of BK7 material. The wedge angle was $3.9^{\circ}$.

\section{Result and discussion.}




\section{3-1. Design of Trepanning Optical System with Two Wedges \\ Let's assume that there is only wedge 1 and wedge 2 in} Figure 1 (a). The angle of wedge 1 and wedge 2 is 3.9o. If the wedge 1 and wedge 2 face each other at $180^{\circ}$, the beam passing through the wedges 1 and 2 generate the offset to the optical axis. When this offset beam passes through the focus lens, it is focused to the specimen with an inclination. In that case, the offset distance from the optical axis increases as the distance between the wedge 1 and wedge 2 increases. When the offset distance increases, the inclination angle irradiated to the specimen increases. Then the focused beam theoretically rotates with only the inclination angle in one point. Figure 3 (a) shows the inclination angle calculated using ray tracing according to distance between wedge 1 and wedge 2. ASAP (Advanced System Analysis Program) is used for ray tracing simulation. For the simulation, BK7 was used as the wedge material. 1 inch size wedge and focus lens of $100 \mathrm{~mm}$ focal length were used. It was assumed that laser source of $6 \mathrm{~mm}$ diameter is irradiated to the wedge.

The focus lens was position at $300 \mathrm{~mm}$ from the wedge 1 . The inclination angle was calculated trigonometrically after first calculating the spot location at $100 \mathrm{~mm}$ from the focus lens and that at $50 \mathrm{~mm}$. As shown in Figure 3 (a), the calculation shows that the inclination angle differs according to the distance between the wedge 1 and wedge 2 . If the distance is $225 \mathrm{~mm}$, the inclination angle can be as big as 4.55 o. large inclination angles makes reverse-tapered drilling possible. In other words, the drilling of higher entrance hole diameter than the exit hole diameter is possible. And the drilling speed increases as the inclination angle increases. It is because there is less reflection and more absorption on the drilled cross section surface and thus the ablation rate is increased. However, the distance between the wedge 1 and wedge 2 must increase to enlarge the inclination angle, and that increases the size of the trepanning optical system. Therefore, it is needed to calculate the optimum inclination angle for the drill application and design the trepanning optical system according to it.

When the wedge 1 and wedge 2 face each other at $180^{\circ}$, the beam with inclination angle will be focused on one point on the specimen. However, if there is the wedge angle difference (manufacturing error) of wedge 1 and wedge 2 , it does not rotate with inclination angle at one point on the specimen, but the focused beam rotates drawing a circle. Let's define the size of circle drawn by the rotating focused beam as trepanning diameter. The trepanning diameter increases as the degree between wedge angle difference between wedge 1 and wedge 2 increases. Figure 3 (b) shows the result of trepanning diameter simulation according to wedge and we angle difference between wedge 1 dge2.

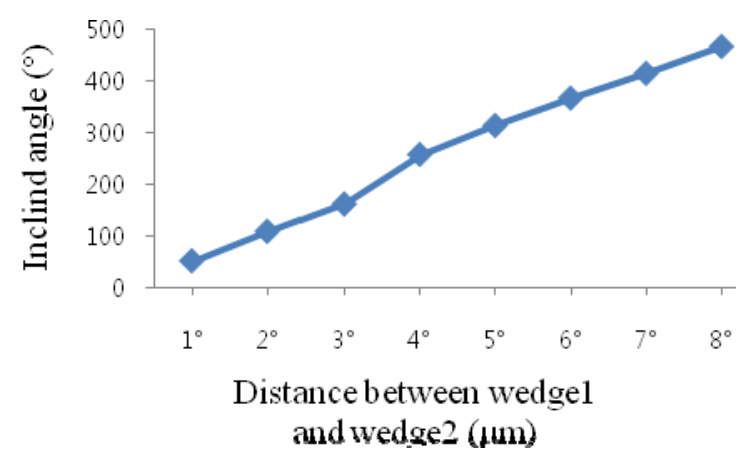

(a)

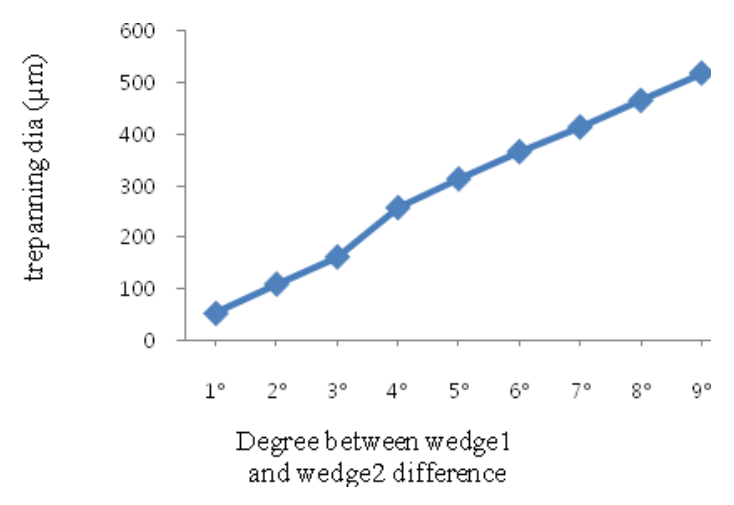

(b)

Figure 3. (a) the ray tracing result of inclined angle according to distance between wedge 1 and wedge 2 .

(b) trepanning diameter according to

difference rotation angle between wedge 1 and wedge 2 .

\section{3-2. Problem and Solution of Trepanning Optical Sys- tem with Two Wedges}

As shown in Figure 3, the angle of beam irradiated to the specimen and trepanning diameter can be controlled using the wedge 1 and wedge 2 . However, it is impossible to independently control the incident angle and trepanning diameter. That is because the trepanning diameter changes when the incident angle changes. If the trepanning diameter is 200um or less, the difference of incident angle is $0.5^{\circ}$ or less. However, the trepanning optical system with two wedges has a problem. If the wedge 1 and wedge 2 are manufactured with $0.1^{\circ}$ difference of wedge angle, it creates trepanning diameter of 104um. For example, if the wedge angle of wedge 1 is $3.9^{\circ}$ and wedge angel of wedge 2 is $4.0^{\circ}$, the trepanning diameter is $104 \mathrm{um}$ even if wedge 1 and wedge 2 face each other at $180^{\circ}$ at accurate alignment. If the spot size of the focused laser is $50 \mathrm{um}$. The total diameter of drilled hole would be $154 \mathrm{um}$. In other words, drilling of holes smaller than 154um would be impossible. Typically, the precision of wedge angle of manufactured wedge is $0.2^{\circ}$ or higher. Therefore, the wedge angle error of $0.2^{\circ}$ is expected even when using the same wedge. Figure 4 shows the trepanning diameter according to difference wedge angle between wedge 1 and wedge 2 . The wedge angle of wedge 2 was changed while that of wedge 1 was fixed at $3.9^{\circ}$. The simulation used the distance of $100 \mathrm{~mm}$ between wedge 1 and wedge 2 , focal length of focus lens at $100 \mathrm{~mm}$, and distance of $100 \mathrm{~mm}$ between the 
focus lens and specimen while the rotation angle of wedge 1 and wedge 2 was fixed at $180^{\circ}$.

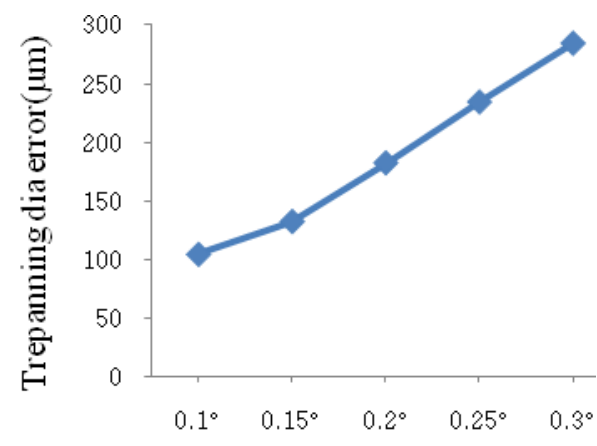

Fabrication error of wedge2

Figure 4 trepanning diameter errors according to difference rotation angle between wedge 1 and wedge 2

In order to compensate the manufacturing error of wedge 1 and wedge 2, wedge 3 and wedge 4 are used in conventional method. Compared to wedge angles of wedges 1 and 2 , those of wedge 3 and 4 are very small. The error generated by wedge 1 and wedge 2 is compensated by wedge 3 and wedge 4 . However, installing wedge 3 and wedge 4 causes the size of trepanning optical system to increase. In this paper, using the tilted focus lens is proposed to maintain the size of trepanning optical system small. As shown in Figure 1 (b), the error generated by wedge 1 and wedge 2 can be compensated using the tilted lens. In other words, the titled lens can reduce the trepanning diameter created, because of manufacturing error of wedge 1 and wedge 2 , even when wedge 1 and wedge 2 face each other at $180^{\circ}$. Figure 5 shows the trepanning diameter according to the tilting angle of focusing lens when the wedge 1 and wedge 2 have $0.1^{\circ}$ difference. As shown in the figure, the trepanning diameter can be controlled by adjusting the tilting angle of the focusing lens.

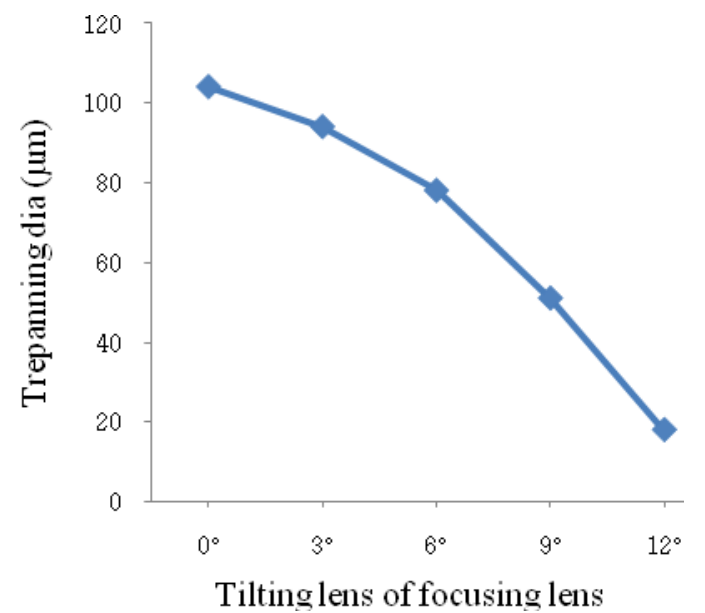

Figure 5. trepanning diameter according to tilting angle of focusing lens.

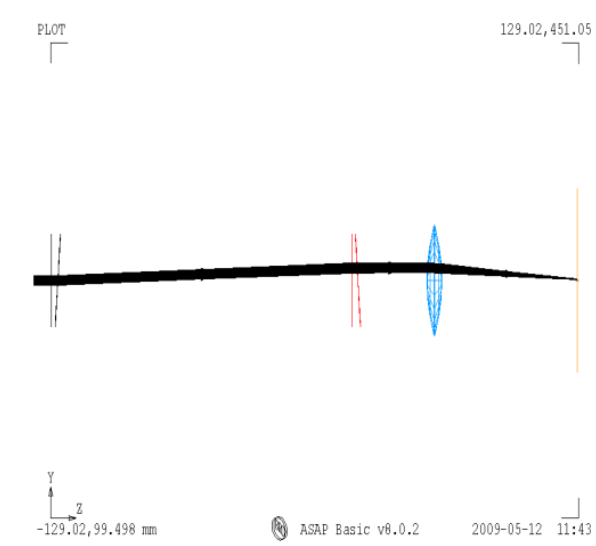

(a)

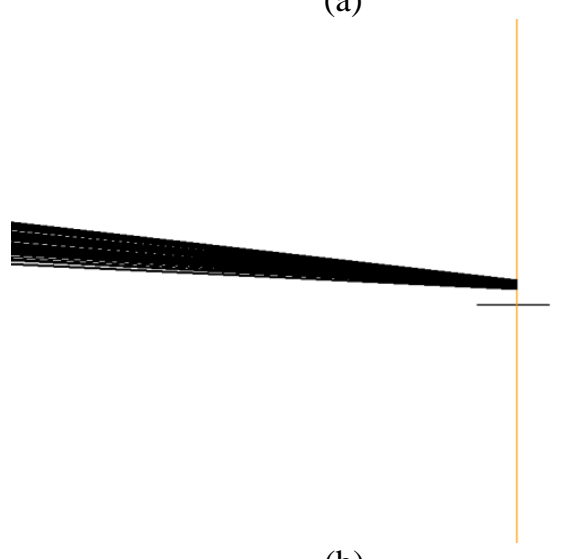

(b)

Figure 6. (a) ray tracing result of two wedge and focusing lens, (b) enlargement of focused plane

Figure 6 (a) shows the result of ray tracing of trepanning optical system with two wedges and a focus lens. The first element from left of Figure 6 (a) represents the wedge 1 while the second element represents the wedge 2 . The third element represents the focusing lens. The right most elements show the drilled surface. The laser beam propagates from left to right. The wedge angle of wedge 1 was $3.9^{\circ}$, and the wedge angle of wedge 2 was assumed to be $3.7^{\circ}$. In other words, the manufacturing error was assumed to be $0.2^{\circ}$. The distance between wedge 1 and wedge 2 was $200 \mathrm{~mm}$ while that between the wedge 2 and focusing lens was $50 \mathrm{~mm}$. The distance between the focusing lens and drilled surface was fixed at $100 \mathrm{~mm}$. Wedge 1 and wedge 2 were positioned so that they face each other exactly at 180 ${ }^{\circ}$. As expected, the beams are not focused on specimen as shown in Figure 6 (b) due to the manufacturing error of $0.2^{\circ}$. The vertically drawn line on the drilled surface in Figure 6 (B) represents the origin. Since the focused beams are not concentrated on the origin, the difference becomes the trepanning diameter. 


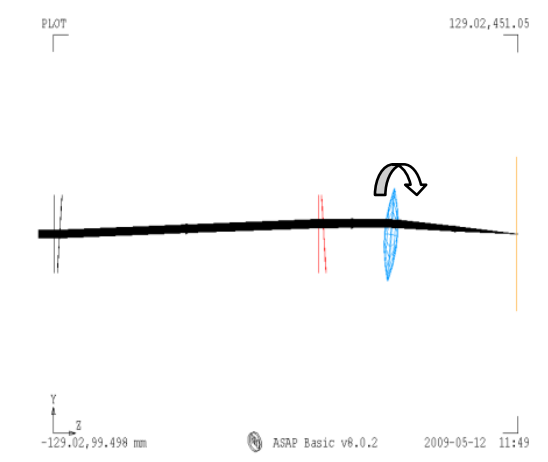

(a)

(b)

Figure 7. (a) ray tracing result of two wedge and tilting focusing lens, (b) enlargement of focused plane

Figure 7 (a) shows the result of ray tracing compensation of error generated by wedge 1 and wedge by tilting the focus lens. All conditions of the simulation were the same as Figure 6 except that the tilting angle of the focus lens was $5^{\circ}$. As shown in Figure 7 (b), the focused beams reach the origin of the drilled surface. The case indicates that the trepanning diameter can be reduced.

\section{3-2. Result of Trepanning Optical Drilling Using Tilted} Focusing Lens

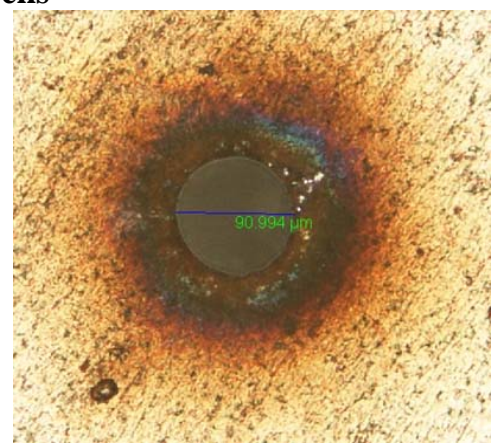

(a)

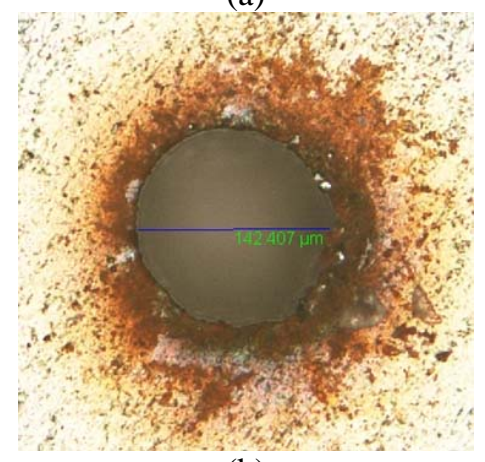

(b)
Figure 8. the microscopy of drilling result using trepanning optics.

Figure 8 shows the result of trepanning optical drilling using tilted focusing lens. Fiber type pulse laser was used. The wavelength of $1064 \mathrm{~nm}$, pulse duration of $80 \mathrm{nsec}$, rep rate of $20 \mathrm{KHz}$ and average power of $10 \mathrm{~W}$ were used. The inclined transmission angle was $2^{\circ}$, and the focusing lens had $100 \mathrm{~mm}$ focal length. The rotation speed of trepanning optical system was 100rpm, and the hole was drilled for 30 seconds. The drilled specimen was 400um thick steel.

Figure 8 (a) shows the case of trepanning diameter fixed at 50um while Figure 8 (b) shows the case of that fixed at 100 um. In both cases, the focused spot diameter was around 40um. Because the nano second laser was used, the heat affection zone existed, but the drilling resulted in relatively clean hole. If the ultra-short laser (picosecond laser or femtosecond laser) was used instead of the nano second laser, drilling would have eliminated more heat affection zone.

\section{Conclusion.}

This paper described the trepanning optical system using wedges for laser drilling. The conventional systems used 4 wedges. Wedges 3 and 4 compensated the manufacturing error of wedges 1 and 2. The trepanning optical system must be mounted in the drill in the actual process. As thus, the trepanning system must be small and light. In this study, the titled focusing lens is used without the wedges 3 and $w$ 4 to compensate the error of wedges 1 and 2 . As the result, the size of the trepanning optical system was reduced.

\section{Acknowledgement}

This work was supported in part by the Korean Ministry of Commerce, Industry and Energy as part of the 'Development of precision-machining technology using advanced lasers' and 'Technology Development of Plastic Injection Molding for Super hydrophobic Surface by Nano-on-Micro Patterns' project

\section{References}

[1] L. Shah, J. Tawney, M. Richardson, and K. Richardson: J. Appl. Surf. Sci., 183, (2001) 151.

[2] S. Juodkazis, H. Okuno, N. Kujime, S. Matsuo, H. Misawa: J. Appl. Phys. A., 79, (2004) 1555.

[3] H. Varel, D. Ashkenasi, A. Rosenfeld, M. Wahmer, E.E.B. Campbell: J. Appl. Phys. A., 65, (1997) 367.

[4] A. Luft, U. Franz, A. Emsermann, J. Kaspar: J. Appl. Phys. A., 63, (1996) 93.

[5] S. Bruneau, J. Hermann, G. Dumitru, M. Sentis, E. Axente: J. Appl. Surf. Sci., 248, (2005) 299

[6] S. M. Klimentov, S. V. Garnov, T. V. Kononenko, V. I. Konov, P. A. Pivovarov, F. Dausinger: J. Appl. Phys. AMater. 69 Suppl., 633. (1999) 636.

[7] P. Christensen, P. Herman, R. Patel (Eds.): LIA 88, LaserInstitute of America, Orlando, (2000) p.195, 203.

[8] I. Miyamoto, Y. F. Lu, K. Sugioka, J. J. Dubowski (Eds.): Proc. SPIE 4426, Intl. Soc. For Opt. Eng., (2002) p. $108,112$. 
[9] C. Föhl, D. Breitling, F. Dausinger: ICALEO, 21st

International Congress on Applications of Laser and Electro-Optics, Congress Proceedings, (2002).

[10] I. Miyamoto, Y.F. Lu, K. Sugioka, J.J. Dubowski

(Eds.): Proc. SPIE 4426, Second Intl. Symposium on Laser Precision Microfabrication, LPM, (2001) p.104, 107.

[11] J.S.Fossa, M.R.B.Andreeta, and A.C.Hernandes :Laser Physics, vol 19 No 10 (2009) pp2045-2049

(Received: July 9, 2009, Accepted: November 18, 2009) 